\title{
LA SUPERVISIÓN DE ENFERMERÍA: CONOCIMIENTOS Y HABILIDADES EN LA FORMACIÓN DE EQUIPOS DE TRABAJO
}

\author{
Karla Sofía Gómez-Alcántara ${ }^{1}$ y Danelia Gómez-Torres² \\ ${ }^{1 y 2}$ Universidad Autónoma del Estado de México. Toluca, México sofia33gom@gmail.com \\ '2gomezdanelia@usa.net
}

\begin{abstract}
Resumen. La supervisión del talento humano de Enfermería es la herramienta para implementar las acciones de provisión de servicios, sin embargo, la complejidad de las características propias del personal genera retos para quienes desarrollan esta función. El objetivo fue identificar la forma en que las supervisoras de enfermería integran equipos de trabajo considerando los conocimientos y habilidades del personal. Material y métodos: se elaboró una guíe de entrevista semiestructurada y se realizó una entrevista personalmente en el área de trabajo de las supervisoras. Se llevó a cabo un análisis descriptivo de los datos y la discusión con la teoría de los roles de equipo de M. Belbin. Resultados: se identificaron las estrategias que las supervisoras utilizan para crear equipos de trabajos con personal que posee diferentes conocimientos y habilidades. Consideraciones finales: se encontró que las supervisoras dan mayor importancia al conocimiento y las habilidades de las enfermeras para integrarlas a un equipo y no su nivel académico.
\end{abstract}

Palabras clave: Supervisión de Enfermería; Enfermería; Conocimientos; Grupo de Enfermería.

\section{NURSING SUPERVISION: KNOWLEDGE AND SKILLS IN THE FORMATION OF WORK TEAMS}

Abstract. The supervision of the human talent of Nursing is the tool to implement the actions of service provision, however, the complexity of the characteristics of the staff creates challenges for those who carry out this function. The objective was to identify the way in which nursing supervisors integrate work teams considering the knowledge and skills of the staff. Material and methods: a semi-structured interview guide was prepared and an interview was conducted in person in the supervisors' work area. A descriptive analysis of the data and discussion with the team role theory of M. Belbin was carried out. Results: The strategies that supervisors use to create work teams with personnel with different knowledge and skills were identified. Final considerations: it was found that the supervisors give greater importance to the knowledge and skills of the nurses to integrate them into a team and not their academic level.

Keywords: Nursing Supervisory; Nursing; Knowledge; Nursing Team.

\section{INTRODUCCIÓN}

Para situar en un contexto general a la supervisión en Enfermería, esta ha sido esencial para desempeñar procesos de gestión dentro de las instituciones hospitalarias, las enfermeras que ejercen la supervisión se enfrentan constantemente a retos en especial en la gerencia del capital humano, ya que por las características propias del personal implica dificultades, sin embargo, la integración de equipos de trabajo ha sido una opción viable para hacerlo (Ministerio de salud, 2014: s/p).

Para dar cumplimento a los procesos de supervisión del talento humano de las unidades hospitalarias, es necesario que las supervisoras apliquen el proceso administrativo, además 
de un amplio conocimiento de gestión del capital humano. Así como la capacidad de comunicación y liderazgo con el personal a su cargo.

Para estudiar el fenómeno de la supervisión se siguió la metodología cualitativa con índole descriptiva, se inició presentando el objetivo general y la pregunta de investigación, para exponer la técnica de recolección de datos, continuando con el análisis de los resultados de la investigación que ofrecen una perspectiva contrastada con otros autores, destacándose los argumentos de las entrevistadas y el referente teórico. Por último se presentan las consideraciones finales.

\section{METODOLOGÍA}

El estudio se planteó con enfoque cualitativo porque se caracteriza por utilizar métodos que intentan capturar el fenómeno de forma holística, entenderlo, comprenderlo dentro de su contexto o enfatizar en la inmersión y compresión del significado humano adscrito a un grupo de circunstancias o fenómeno, o las tres cosas (Lincoln, 2013: 58).

Fue de un nivel descriptivo, porque buscó especificar las propiedades importantes de las personas y el fenómeno que se sometió a análisis, además se seleccionó una serie de cuestiones y se mencionó cada una de ellas independientemente, de forma tal, describir lo que se investiga (Cauas, 2015: 2). Así mismo, permitió caracterizar un fenómeno concreto, indicando sus rasgos peculiares, describiendo la forma en qué los supervisores integran equipos de trabajo, sin intervención ni manipulación de variables.

La investigación tuvo como objeto de estudio: conocer las estrategias que las supervisoras aplican para integrar en equipos de trabajo a personal con diferentes características de conocimientos y habilidades, en una unidad hospitalaria, teniendo como pregunta de investigación ¿Cuáles son las estrategias que las supervisoras aplican para integrar en equipos de trabajo a personal con diferentes características de conocimientos y habilidades, en una unidad hospitalaria?

El proceso adoptado para la recolección de datos fue el método de la entrevista individual, apoyada por una guía semi-estructurada con once preguntas abiertas, las cuales surgieron de preguntas conductoras a partir de la teoría de los roles de equipo de M. Belbin. Se consideraron como criterios de inclusión: contar con un mínimo de dos años de experiencia profesional en el cargo desempeñado, con trayectoria que acreditara su formación 
profesional en el área administrativa y tener un posgrado académico, así mismo fungir como supervisoras de enfermería. Como criterios de exclusión serian aquellas supervisoras de enfermeras que no desearan participar en la investigación y, se eliminaron dos entrevistas que no aportaron datos relevantes a la investigación, siendo un total de 10 entrevistadas, pues con ese número de entrevistadas se logró la saturación de datos, de acuerdo con los objetivos de la investigación, tal y como lo determina la metodología cualitativa

Las entrevistas fueron realizadas de un escenario con iluminación, libre de ruidos y de otro tipo de distractores y se grabaron en audio con previa revisión de los instrumentos de grabación para verificar su óptima funcionabilidad. El audio de las entrevistas se escuchó en varias ocasiones y posteriormente fueron transcritas en su totalidad por las investigadoras.

Para el análisis y tratamiento de los datos obtenidos de las entrevistas, se utilizó la técnica cromática, que consiste en tener los datos recolectados y transcritos, se leyeron por más de una vez para realizar la selección de fragmentos que se clasificaron en las pre categorías correspondientes a la investigación, mediante distintos colores se aglutinaron los datos o hallazgos, se pre etiquetaron en una gama de colores para ser analizados, e incluso determinar sí surgían o no nuevas categorías.

La discusión de los resultados se efectuó contrastando datos empíricos con el referencial teórico, con la teoría de los roles de equipo de M. Belbin. En conclusión se buscó demostrar que los hallazgos presentados eran los más sobresalientes de la investigación buscando fueran estos los que generasen los fundamentos, para la conformación de las estrategias en la creación de equipos de trabajo. Los resultados obtenidos se presentaron en subcapítulos según las categorías que emergieron.

\section{RESULTADOS Y DISCUSIÓN}

Los resultados obtenidos en esta investigación, se contrastaron con la teoría de los roles de equipo de M. Belbin, la cual pretende conocer la manera de formar equipos ideales dentro de las organizaciones, mediante la combinación adecuada de los roles funcionales y de equipo, es decir, considerando el perfil de los individuos, haciéndola aplicable al problema en estudio, de esta manera se logró proyectar como las supervisoras integran los equipos de trabajo en Enfermería, los cuales se describen en categorías y subcategorías. 


\subsection{Las habilidades técnicas}

Las habilidades técnicas se refieren a la capacidad que tienen las personas para aplicar métodos, procedimientos y técnicas de un área del conocimiento específica y que pueden ser especializadas (ITESM, 2017: s/p). En los equipos de trabajo se hace uso de las habilidades técnicas de los integrantes para alcanzar los objetivos del equipo, de ahí la importancia de identificarlas y desarrollarlas tal como lo refieren las entrevistadas:

Identificar las habilidades con las que cuentan pues esto les facilita mucho adaptarse a los servicios (E-8).

Las habilidades son muy importantes porque esto hará que el equipo funcione (E-5).

Estas habilidades además implican talento para realizar determinadas tareas, sin embargo, no significa que se tenga aptitud para realizar otro tipo de funciones, de ahí que el desarrollo de la habilidad es una parte de competencia para cumplir con una meta específica (ITESM, 2017: s/p). Para que las habilidades técnicas del personal de Enfermería sean empleadas de manera óptima en la conformación de los equipos de trabajo las supervisoras implementan algunas estrategias:

Procuro que haya personas novatas y personas expertas, porque así va a haber la diferencia de cambio [complementariedad] (E-1).

Las habilidades técnicas influyen en el rol que cada integrante del equipo desempeña, pues esto lo puede hacer experto en un área, siendo parte de las fortalezas que puede aportar al equipo y debe existir una potenciación continua de las mismas. Es importante que los gestores de los equipos sepan que quienes fracasan en un equipo pueden triunfar en otro (Belbin, 2008: 55), las habilidades facilitan la adaptación a los equipos y mejoran los resultados.

Lo anterior muestra que las habilidades técnicas de las enfermeras son indispensables para que se integren a un equipo especifico, en el cual se puedan emplear sus habilidades para el logro de objetivos, así mismo se requiere que el personal se complemente con los demás integrantes, ya que cada uno posee habilidades distintas que finalmente harán funcional al equipo y lo llevaran a conseguir sus metas, lo cual se reflejará en la calidad de atención a los pacientes. 


\subsection{Los conocimientos}

El conocimiento es un cumulo de información interpretada en la realidad que la persona posee, puede ser adquirido de forma científica o empírica, Enfermería se reconoce por ser una disciplina profesional cuya esencia es la práctica sustentada en el conocimiento científico. Este conocimiento científico se fundamenta en el conocimiento propio de enfermería derivado de los resultados de su investigación, y del conocimiento teórico propio y de otras disciplinas aplicado y adaptado a la práctica, que conforman la esencia disciplinar (Durán, 2018: 58). El conocimiento se ha considerado en los últimos años como un recurso indispensable para la producción de servicios, tal como se refleja en las respuestas de las supervisoras:

Los conocimientos son muy importantes porque a través de ellos, ellas [las enfermeras] se apegan a la normatividad, aplican los procesos de enfermería cómo deben de ser (E-5).

En la gerencia actual debido a su importancia requiere de los conocimientos que la enfermera adquiere en la formación, siendo necesario el poseer conocimientos en el campo de la gestión, se puede afirmar que las personas son valoradas por sus conocimientos, sus aportes intelectuales y talento en la consecución de las metas de las organizaciones (Majad, 2016: 149), es por eso que las supervisoras de enfermería aplican estrategias que permiten fundamentar las acciones en los conocimientos del personal:

Elegimos al personal que tiene conocimientos específicos de un servicio (...) para aprovechar las potencialidades de las enfermeras, así lograr hacer bien el trabajo y eso repercute directamente en la atención a los pacientes (E-8).

En los equipos de trabajo la solución denominada tanque de inteligencia es la manera ideal de aprovechar el talento humano y utilizarlo eficazmente en campos fundamentales. En el ámbito de gestión donde existen muchas reglas complejas, limitaciones, variables y posibilidades se crea una situación donde se cotiza mucho el conocimiento e inteligencia (Belbin, 2000: 12). Sin embargo, el personal que desempeña este rol no encaja fácilmente en un sistema y requieren de una persona que les dirija: un jefe ideal que tenga la habilidad de descubrir el talento de estas personas y saber cómo y cuándo utilizarlo, además un jefe comprensivo y amable sacará a la luz lo mejor de las personas con alto nivel de conocimientos (Belbin, 2001: 69).

Es así que el conocimiento que posee el personal de Enfermería es indispensable para desempeñarse dentro de un área específica, pero además es fundamental que dentro de un 
equipo de trabajo los integrantes realicen la práctica sustentada en el conocimiento científico, sin embargo, el éxito de este tipo de equipos depende en gran medida de la capacidad de las supervisoras para identificar y saber utilizar el conocimiento de su personal.

\subsection{El grado académico}

Se entiende por grado académicos como un título académico otorgado por una universidad o colegio y es significado de que se ha completado un programa académico (Mendoza, 2015: 282). En la actualidad el tener grados académicos más allá de dar un licenciamiento para ejercer una profesión determinada, implica tener el dominio de la misma, dando lugar a la expertez, un atributo que se les da a las personas que demuestran el dominio del conocimiento y práctica, tal como lo refiere la supervisora entrevistada:

Nos damos cuenta con qué nivel académico vienen y eso puede servir de fortaleza para reforzar equipos en zonas críticas del hospital (E-8).

En la integración de equipos de trabajo, el grado académico representa complejidad, ya que en Enfermería aún existe personal sub profesional laborando en las instituciones de salud y el personal con grado académico es muy variado; desde licenciadas hasta doctoras, es por eso que las supervisoras emplean una serie de estrategias para realizar la composición de equipos de trabajo:

Yo manejo la fortaleza de personal si eres pediatra pues lógico es que te voy a mandar pediatría, si eres intensivista te mando terapia o a medicinas internas (E-6).

Tal como se refleja en las expresiones anteriores, el rol laboral de un persona en la vida ya no está definido por la edad, el sexo o la raza, sino por la educación y experiencia profesional, aquellos que cumplen con estos factores son muy apreciados, el título profesional se convirtió en la descripción de la persona ya que así las personas se identifican así mismas en términos de lo que han aprendido y para lo que están cualificadas, es decir, que un título profesional proclama tanto la ocupación como la identidad (Belbin, 2001: 15).

De tal manera que los grados académicos son una fortaleza en función de la expertez que tienen las personas altamente cualificadas para desempeñarse profesionalmente, no obstante, tener a personas con grados académicos altos en equipos de Enfermería no siempre asegura su éxito, se requiere que las enfermeras se sientan identificadas con su 
profesión y que desempeñen roles distintos, aun cuando todas tuvieran el mismo grado académico, estas tendrán características personales que deben ser analizadas para que se complementen como equipo.

\section{CONSIDERACIONES FINALES}

La investigación permitió mediante el análisis crítico y reflexivo obtener de los resultados dar respuesta a la interrogante: ¿Cuáles son las estrategias que las supervisoras aplican para integrar en equipos de trabajo a personal con diferentes características de conocimientos y habilidades, en una unidad hospitalaria?, tales elementos se integran en tres grupos: habilidades técnicas, conocimientos y grado académico del personal de enfermería.

Con resultados obtenidos se consiguió mostrar y comprender que la enfermera supervisora realiza funciones administrativas y gerenciales para integrar equipos de trabajo, para esto aplica el proceso administrativo, sin embargo, se encontró que la supervisora también emplea sus habilidades para la identificación de características cualitativas de su personal, lo que permite mejorar sus estrategias para la gestión del talento humano.

No obstante, también se encontró que las supervisoras dan mayor importancia al conocimiento y las habilidades de las enfermeras para integrarlas a un equipo y no su nivel académico, tal como lo que refiere la bibliografía, lo cual les crea dificultades al no contar con personal que cumpla el perfil deseado para desempeñar las funciones de los organigramas.

Un hallazgo sobresaliente es que le otorgan un valor importante a la comunicación dentro de los equipos, porque esta les permite transmitir información así los integrantes trabajan colaborativamente, además se evitan conflictos, favoreciendo la convivencia, también identifican rasgos de la personalidad de las enfermeras para integrarlas en equipos donde les sea fácil su adaptación.

Un aspecto a destacar es que se logró realizar un muestreo apropiado al seleccionar a participantes que tienen conocimientos específicos de la supervisión pues con esto se logró una saturación de datos efectiva de las categorías, con información de calidad. Además, el enfoque cualitativo de la investigación permitió comprender el proceso de formación de los equipos, aun cuando la supervisión en enfermería fue un fenómeno complejo de estudiar. Se consideraron en todo momento los objetivos de la investigación para seguir la línea del 
rigor metodológico cualitativo, evitando errores de interpretación de los hechos, con la contratación de datos con otros autores. Por lo cual se obtuvieron resultados fiables y de relevancia científica para la profesión de Enfermería

\section{REFERENCIAS}

Belbin, M. (1981). Managment teams: Way the succeed or fail. Oxford: Butterwotrh-Heineman.

Belbin, M. (2012). Beyond the team. Oxford: Butterwotrh-Heineman.

Belbin, M. (2000a). Equipos directivos: el por qué de su éxito y fracaso. Inglaterra: Belbins.

Belbin, M. (2000). La guía de Belbin para triunfar en el trabajo. Inglaterra: Belbins.

Belbin, M. (2001).Roles de equipo en el trabajo. Inglaterra: Belbins.

Cauas, D. (2015). Definición de las variables, enfoque y tipo de investigación. Biblioteca electrónica de la Universidad Nacional de Colombia, (1), 11.

Durán de Villalobos, M. (2018). Una mirada hacia afuera: proyección de enfermería para el 2050. Aquichan, 18(1), 5-8. Disponible en: http://www.redalyc.org/articulo.oa?id=74155410001

Instituto Tecnológico y de Estudios Superiores de Monterrey. (2017). El desarrollo de habilidades, valores y actitudes propuestos en la misión. Disponible en: http://sitios.itesm.mx/va/dide2/documentos/havs.pdf

Lincoln, I. (2013). Conexiones afines entre los métodos cualitativos y la investigación en salud. Revista Investigación y educación en Enfermería, Vol. 15, 57-69.

Majad Rondón, M. (2016). Gestión del talento humano en organizaciones educativas. Revista de Investigación, 40(88), 148-165. Disponible en: http://www.redalyc.org/articulo.oa?id=376147131008

Mendoza, P. (2015). Los Grados Académicos: Surgimiento y Evolución. Anales de la Facultad de Medicina, 61(4), 278-284. Disponible en: http://www.redalyc.org/articulo.oa?id=37961403

Ministerio de Salud (2014). Lineamientos técnicos para la supervisión de enfermería en las RIISS. Disponible en: http://asp.salud.gob.sv/regulacion/pdf/lineamientos/lineamientos_tecnicos_supervision_enfermeria_riiss_31 012014.pdf 